\title{
Non-invasive liver fibrosis assessment and HCV treatment initiation within a systematic screening program in HIV/HCV coinfected patients
}

\author{
David Chromy (D) Philipp Schwabl · Theresa Bucsics • Bernhard Scheiner · Robert Strassl • Florian Mayer • \\ Maximilian C. Aichelburg · Katharina Grabmeier-Pfistershammer - Michael Trauner • Markus Peck- \\ Radosavljevic · Thomas Reiberger · Mattias Mandorfer
}

Received: 22 May 2017 / Accepted: 26 June 2017 / Published online: 25 July 2017

(C) The Author(s) 2017. This article is an open access publication.

\begin{abstract}
Summary
Background and aim Hepatitis C virus (HCV) therapy should be considered without delay in all patients with significant (SIGFIB) or advanced liver fibrosis (ADVFIB). We aimed to investigate the rates of treatment initiation with interferon-free regimens within a screening program for SIGFIB/ADVFIB in human immunodeficiency virus/HCV coinfected patients (HIV/HCV).

Methods The FIB-4 was calculated in all HIV/HCV from 2014-2016. HIV/HCV were counselled by the HIV clinic and referred to the Division of Gastroen-
\end{abstract}

Electronic supplementary material The online version of this article (doi: 10.1007/s00508-017-1231-x) contains supplementary material, which is available to authorized users.

D. Chromy · P. Schwabl, M.D. · T. Bucsics, M.D.

B. Scheiner, M.D. · M. Trauner, M.D.

M. Peck-Radosavljevic, M.D. · T. Reiberger, M.D. ·

M. Mandorfer, M.D. ( $ه)$

Division of Gastroenterology and Hepatology, Department

of Internal Medicine III, Medical University of Vienna,

Währinger Gürtel 18-20, 1090 Vienna, Austria

mattias.mandorfer@meduniwien.ac.at

D. Chromy · P. Schwabl, M.D. · T. Bucsics, M.D. ·

B. Scheiner, M.D. • M. C. Aichelburg, M.D.

K. Grabmeier-Pfistershammer, M.D. .

M. Peck-Radosavljevic, M.D. · T. Reiberger, M.D.

M. Mandorfer, M.D.

Vienna HIV \& Liver Study Group, Medical University of

Vienna, Vienna, Austria

R. Strassl, M.D. · F. Mayer, M.D.

Department of Laboratory Medicine, Division of Clinical

Virology, Medical University of Vienna, Vienna, Austria

M. C. Aichelburg, M.D. · K. Grabmeier-Pfistershammer, M.D. Division of Immunology, Allergy and Infectious Diseases, Department of Dermatology, Medical University of Vienna, Vienna, Austria terology and Hepatology for transient elastography (TE) and evaluation for HCV therapy. Patients were stratified by FIB-4 of $</ \geq 1.45$ (established cut-off for ruling out ADVFIB) and SIGFIB/ADVFIB were defined by liver stiffness $>7.1 \mathrm{kPa} />9.5 \mathrm{kPa}$, respectively. Results Among $1348 \mathrm{HIV}+$ patients, 16\% (210/1348) had detectable HCV-RNA. One hundred HIV/HCV had a FIB- $4 \geq 1.45$. Among these, 57\% (57/100) underwent TE. The majority of these patients had SIGFIB $(75 \%$; $43 / 57)$ or ADVFIB (37\%; 21/57), however, interferonfree treatment was initiated in only 56\% (24/43).

In addition, fifty-two percent (57/110) of HIV/HCV with FIB-4 $<1.45$ underwent TE. Interestingly, $40 \%$ $(23 / 57)$ and $18 \%(10 / 57)$ of these patients showed SIGFIB or even ADVFIB, respectively, and 78\% (18/23) finally received interferon-free treatment. Overall, only $20 \%(42 / 210)$ of $\mathrm{HIV} / \mathrm{HCV}$ received interferonfree treatment.

Conclusion FIB-4 was not useful for ruling out SIGFIB/ADVFIB in our cohort of HIV/HCV. Treatment was initiated only in a small proportion $(20 \%)$ of $\mathrm{HIV} / \mathrm{HCV}$ during the first 2 years of interferon-free treatment availability, although the observed proportion of patients with SIGFIB (assessed by TE) was considerably higher $(58 \%)$. Thus, it requires the ongoing combined efforts of both HIV and HCV specialists to increase treatment uptake rates in this special population.

Keywords HIV · Hepatitis C · Liver Cirrhosis · Elasticity Imaging Techniques

\section{Introduction}

Worldwide, approximately 80 million people suffer from chronic hepatitis $\mathrm{C}(\mathrm{CHC})[1]$. In low prevalence areas like Central and Western Europe the main route of transmission is intravenous drug abuse (IVDA) [2], 
which is also associated with a high risk of human immunodeficiency virus/hepatitis $\mathrm{C}$ virus (HIV/HCV) coinfection [3, 4]. In addition, there is an ongoing epidemic of acute hepatitis C among HIV-positive men who have sex with men (MSM) [5]. With $85 \%$ of acute HCV infections in HIV-positive patients resulting in $\mathrm{CHC}$ [6], the prevalence of $\mathrm{CHC}$ among HIVpositive persons in Western Europe and the USA is estimated to be as high as $25-30 \%$ [2]. An HIV/HCV coinfection is associated with accelerated progression towards advanced liver disease as compared to HCV monoinfections [7] and thus, making HCV-associated liver disease a major contributor to morbidity and mortality in HIV-positive patients [8]; however, novel interferon-free (IFN-free) directly acting antiviral (DAA) regimens have largely improved sustained virological response (SVR) rates in $\mathrm{HIV} / \mathrm{HCV}$ coinfection: following the promising results of the initial studies using sofosbuvir (SOF) and ribavirin (RBV) [9], several studies investigating second generation DAA combination regimens, such as SOF/daclatasvir (DCV) [10], SOF/ledipasvir (LDV) [11], ombitasvir/ ritonavir-boosted paritaprevir \pm dasabuvir $(2 \mathrm{D} / 3 \mathrm{D})$ [12], and grazoprevir (GZV)/elbasvir (EBV) [13, 14] reported excellent SVR rates exceeding 95\%. According to the European Association for the Study of the Liver (EASL) recommendations [15], the treatment indications for $\mathrm{HIV} / \mathrm{HCV}$ coinfected patients are identical to HCV monoinfections. While all patients with $\mathrm{CHC}$ should be considered for antiviral therapy, treatment should not be delayed in patients with significant fibrosis (SIGFIB, i.e. METAVIR $\geq$ fibrosis stage 2). Nevertheless, in many countries including Austria reimbursement of IFN-free treatment is commonly restricted to patients with SIGFIB or patients infected with a specific HCV genotype due to its substantial impact on the health insurance budget [16]. Thus, assessment of the severity of liver fibrosis is crucial for treatment initiation. The most widely used noninvasive method for the assessment of liver fibrosis is transient elastography (TE), which is accepted as a surrogate of liver fibrosis and substitute for invasive liver biopsy by the Austrian health insurances [17].

The FIB-4, an index based on simple laboratory parameters, e.g. age, aspartate aminotransferase (AST), platelet count (PLT), alanine aminotransferase (ALT) has been developed to rule out (FIB- 4 1.45: negative predictive value, NPV: $90 \%$ ) or include (FIB-4 $>3.25$ : positive predictive value, PPV: $65 \%$ ) advanced fibrosis (ADVFIB, i. e. METAVIR $\geq \mathrm{F} 3$ ) in $\mathrm{HIV} / \mathrm{HCV}$ coinfected patients [18] and has been extensively validated against liver biopsies [19]. Importantly, FIB-4 is readily available and can thus be easily applied in cohorts of HIV/HCV coinfected to identify patients with increased risk for SIGFIB or ADVFIB and in whom treatment should not be delayed.

Within this systematic screening project, HIV/HCV coinfected patients were counselled by the HIV clinic and referred to the Division of Gastroenterology and Hepatology for TE and evaluation for HCV therapy.

The study aimed to explore whether FIB-4 index is an accurate tool to identify patients who are at considerable risk for SIGFIB or ADVFIB, and thus, might have an urgent need for antiviral therapy. Moreover, the proportions of patients with DDIs between DAAbased regimens and antiretroviral therapy (ART) as well as the rates of treatment uptake in a cohort of $\mathrm{HIV} / \mathrm{HCV}$ coinfected patients were assessed.

\section{Methods}

\section{Study design and population}

Within this systematic screening project, HIV/HCV coinfected patients were counselled by the HIV clinic and referred to the Division of Gastroenterology and Hepatology for TE and evaluation for HCV therapy between 2014 and 2016. All HIV-positive patients with chronic hepatitis $\mathrm{C}$ were included in this retrospective analysis of the screening program.

\section{Assessed parameters}

Epidemiological characteristics were assessed from patient medical history. The HCV genotype was determined using the VERSANT ${ }^{\circledR}$ HCV Genotype 2.0 Assay Line Probe Assay (LiPA, Siemens Healthcare Diagnostics, Tarrytown, NY) and HCV-RNA was assessed using the Abbott RealTime HCV assay (Abbott Molecular, Des Plaines, IL) with a lower limit of quantification and detection of $12 \mathrm{IU} \mathrm{ml}^{-1}$.

\section{FIB-4 and liver stiffness measurement}

Measurement of liver stiffness was performed by TE (Fibroscan ${ }^{\circledR}$, Echosens, Paris, France), as previously described $[20,21]$. The FIB-4 was calculated as age (years) $\times$ AST $\left.(\mathrm{U} / \mathrm{l}) \times \operatorname{PLT}\left(10^{9} / \mathrm{l}\right) \times \operatorname{ALT}(\mathrm{U} / \mathrm{l})^{1 / 2}\right)^{-1}[18]$ :

The SIGFIB and ADVFIB were defined by liver stiffness values $>7.1$ and $>9.5 \mathrm{kPa}$, respectively [22]. $\mathrm{Pa}-$ tients were stratified according to FIB-4 of 1.45, a previously established cut-off for ruling out ADVFIB [23].

\section{Statistical analysis}

Statistical analyses were performed using IBM SPSS Statistics 23 (SPSS, Armonk, NY, USA). Initially, normal distribution of continuous variables was tested by applying the Kolmogorov-Smirnov test to both the entire study population and to each individual subgroup. Continuous variables were reported as mean \pm standard deviation or median (interquartile range), while categorical variables were reported as number of patients with/without (proportion of patients with) the certain characteristics.

Student's t-test was used for group comparisons of continuous variables when applicable. Otherwise, the 
Table 1 Comparison of HIV/HCV-coinfected patients who underwent liver fibrosis assessment by transient elastography (TE) vs. patients who did not

\begin{tabular}{|c|c|c|c|c|}
\hline Patient characteristics & $\begin{array}{l}\text { All patients } \\
(n=210)\end{array}$ & $\begin{array}{l}\text { Underwent TE } \\
(n=114)\end{array}$ & $\begin{array}{l}\text { Without TE } \\
(n=96)\end{array}$ & $P$-value \\
\hline \multicolumn{5}{|l|}{ Epidemiological characteristics } \\
\hline \multicolumn{5}{|l|}{ Sex } \\
\hline Male & $65 \%(136 / 210)$ & $68 \%(77 / 114)$ & $61 \%(59 / 96)$ & \multirow[t]{2}{*}{0.358} \\
\hline Female & $35 \%(74 / 210)$ & $32 \%(37 / 114)$ & $39 \%(37 / 96)$ & \\
\hline Age & $39.1 \pm 10.8$ & $41.5 \pm 11.0$ & $36.3 \pm 10.0$ & $<0.001$ \\
\hline \multicolumn{5}{|l|}{ Transmission } \\
\hline MSM & $5 \%(10 / 210)$ & $7 \%(8 / 114)$ & $2 \%(2 / 96)$ & \multirow[t]{4}{*}{0.233} \\
\hline IVDU & $75 \%(158 / 210)$ & $71 \%(81 / 114)$ & $80 \%(77 / 96)$ & \\
\hline Heterosexual & $14 \%(30 / 210)$ & $17 \%(19 / 114)$ & $12 \%(11 / 96)$ & \\
\hline Others & $6 \%(12 / 210)$ & $5 \%(6 / 114)$ & $6 \%(6 / 96)$ & \\
\hline \multicolumn{5}{|l|}{ Laboratory parameters } \\
\hline Hemoglobin $\left(\mathrm{g} \mathrm{dl}^{-1}\right)$ & $13.8(2.55)$ & $13.8(2.25)$ & $13.6(2.90)$ & 0.066 \\
\hline Platelet count $\left(10^{9} \mathrm{I}^{-1}\right)$ & $196(96.3)$ & $195(94)$ & $199(102)$ & 0.731 \\
\hline White blood cell count $\left(10^{9} \mathrm{I}^{-1}\right)$ & $6.42(3.35)$ & $6.45(3.38)$ & $6.28(3.36)$ & 0.900 \\
\hline Prothrombin time $(\%)$ & $95.4 \pm 25.4$ & $97.3 \pm 25.4$ & $92.9 \pm 25.1$ & 0.249 \\
\hline Albumin $\left(\mathrm{g} \mathrm{dl}^{-1}\right)$ & $42.7(6)$ & $43.2(5.8)$ & $42.0(7.2)$ & $<0.001$ \\
\hline Creatinine $\left(\mathrm{mg} \mathrm{dl}^{-1}\right)$ & $0.82(0.26)$ & $0.84(0.24)$ & $0.81(0.30)$ & 0.189 \\
\hline Bilirubin $\left(\mathrm{mg} \mathrm{dl}^{-1}\right)$ & $0.50(0.4)$ & $0.50(0.46)$ & $0.49(0.39)$ & 0.524 \\
\hline AST $\left(U I^{-1}\right)$ & $44.0(31)$ & $43.5(27.3)$ & $47.0(35.5)$ & 0.474 \\
\hline $\operatorname{ALT}\left(\mathrm{U} \mathrm{I}^{-1}\right)$ & $40.5(42.8)$ & $41.0(28.5)$ & $38.5(58.0)$ & 0.847 \\
\hline $\mathrm{GGT}\left(\mathrm{U} \mathrm{I}^{-1}\right)$ & $76.0(88.0)$ & $76.0(86.0)$ & $70.5(96.3)$ & 0.973 \\
\hline \multicolumn{5}{|l|}{ HIV infection parameters } \\
\hline CD4+ T-lymphocyte count (cells $\mu{ }^{-1}$ ) & $455(368)$ & $511(414)$ & $352(380)$ & 0.001 \\
\hline HIV-RNA $<50$ copies $\mathrm{ml}^{-1}$ & $63 \%(130 / 207)$ & $74 \%(84 / 113)$ & $49 \%(46 / 94)$ & $<0.001$ \\
\hline HIV-RNA $<400$ copies $\mathrm{mr}^{-1}$ & $76 \%(157 / 207)$ & $89 \%(100 / 113)$ & $61 \%(57 / 94)$ & $<0.001$ \\
\hline CART & $91 \%(190 / 210)$ & $95 \%(108 / 114)$ & $85 \%(82 / 96)$ & 0.022 \\
\hline $\mathrm{PI}$ & $57 \%(108 / 190)$ & $44 \%(48 / 108)$ & $73 \%(60 / 82)$ & $<0.001$ \\
\hline$N(t) R T I$ & $93 \%(177 / 190)$ & $94 \%(102 / 108)$ & $92 \%(75 / 82)$ & 0.420 \\
\hline NNRTI & $18 \%(35 / 190)$ & $21 \%(23 / 108)$ & $15 \%(12 / 82)$ & 0.241 \\
\hline II/EI & $25 \%(47 / 190)$ & $35 \%(38 / 108)$ & $11 \%(9 / 82)$ & $<0.001$ \\
\hline \multicolumn{5}{|l|}{ HCV infection parameters } \\
\hline HCV-RNA $\left(\log I U \mathrm{ml}^{-1}\right)$ & $5.93(1.40)$ & $6.00(1.07)$ & $5.75(2.02)$ & 0.214 \\
\hline HCV genotype & $87 \%(183 / 210)$ & $94 \%(107 / 114)$ & $79 \%(76 / 96)$ & - \\
\hline 1 & $57 \%(104 / 183)$ & $56 \%(60 / 107)$ & $58 \%(44 / 76)$ & \multirow[t]{4}{*}{0.123} \\
\hline 2 & $1 \%(3 / 183)$ & $0 \%(0 / 107)$ & $4 \%(3 / 76)$ & \\
\hline 3 & $32 \%(58 / 183)$ & $32 \%(34 / 107)$ & $32 \%(24 / 76)$ & \\
\hline 4 & $10 \%(18 / 183)$ & $12 \%(13 / 107)$ & $6 \%(5 / 76)$ & \\
\hline \multicolumn{5}{|l|}{ Liver stiffness } \\
\hline $\mathrm{F} 0 / \mathrm{F} 1(<7.1 \mathrm{kPa})$ & - & $42 \%(48 / 114)$ & - & - \\
\hline F2 ( $\geq 7.1$ and $<9.5 \mathrm{kPa})$ & - & $31 \%(35 / 114)$ & - & - \\
\hline $\mathrm{F} 3(\geq 9.5$ and $<12.5 \mathrm{kPa})$ & - & $7 \%(8 / 114)$ & - & - \\
\hline $\mathrm{F} 4(\geq 12.5 \mathrm{kPa})$ & - & $20 \%(23 / 114)$ & - & - \\
\hline Significant liver fibrosis ( $\geq 7.1 \mathrm{kPa}$ ) & - & $58 \%(66 / 114)$ & - & - \\
\hline Advanced liver fibrosis ( $\geq 9.5 \mathrm{kPa}$ ) & - & $27 \%(31 / 114)$ & - & - \\
\hline FIB- $4<1.45$ & $52 \%(110 / 210)$ & $50 \%(57 / 114)$ & $55 \%(53 / 96)$ & \multirow[t]{2}{*}{0.452} \\
\hline FIB-4 $\geq 1.45$ & $48 \%(100 / 210)$ & $50 \%(57 / 114)$ & $45 \%(43 / 96)$ & \\
\hline
\end{tabular}


Fig. 1 Proportions of patients undergoing liver fibrosis assessment by transient elastography (TE) and HCV treatment stratified by FIB-4 index. Of the HIV/HCV coinfected patients 100 had a FIB- $4 \geq 1.45$ (right panel). Among these, 57\% (57/100) underwent TE. The majority of these patients had significant (SIGFIB, 75\%; 43/57) or advanced liver fibrosis (ADVFIB, 37\%; 21/57), however, interferon-free treatment was initiated in only $56 \%(24 / 43)$. In addition, $52 \%(57 / 110)$ of HIV/HCV coinfected patients with FIB$4<1.45$ underwent TE (left panel). Interestingly, $40 \%$ (23/57) and $18 \%(10 / 57)$ of these patients showed SIGFIB or even ADVFIB, respectively, and $78 \%$ (18/23) finally received interferon-free treatment

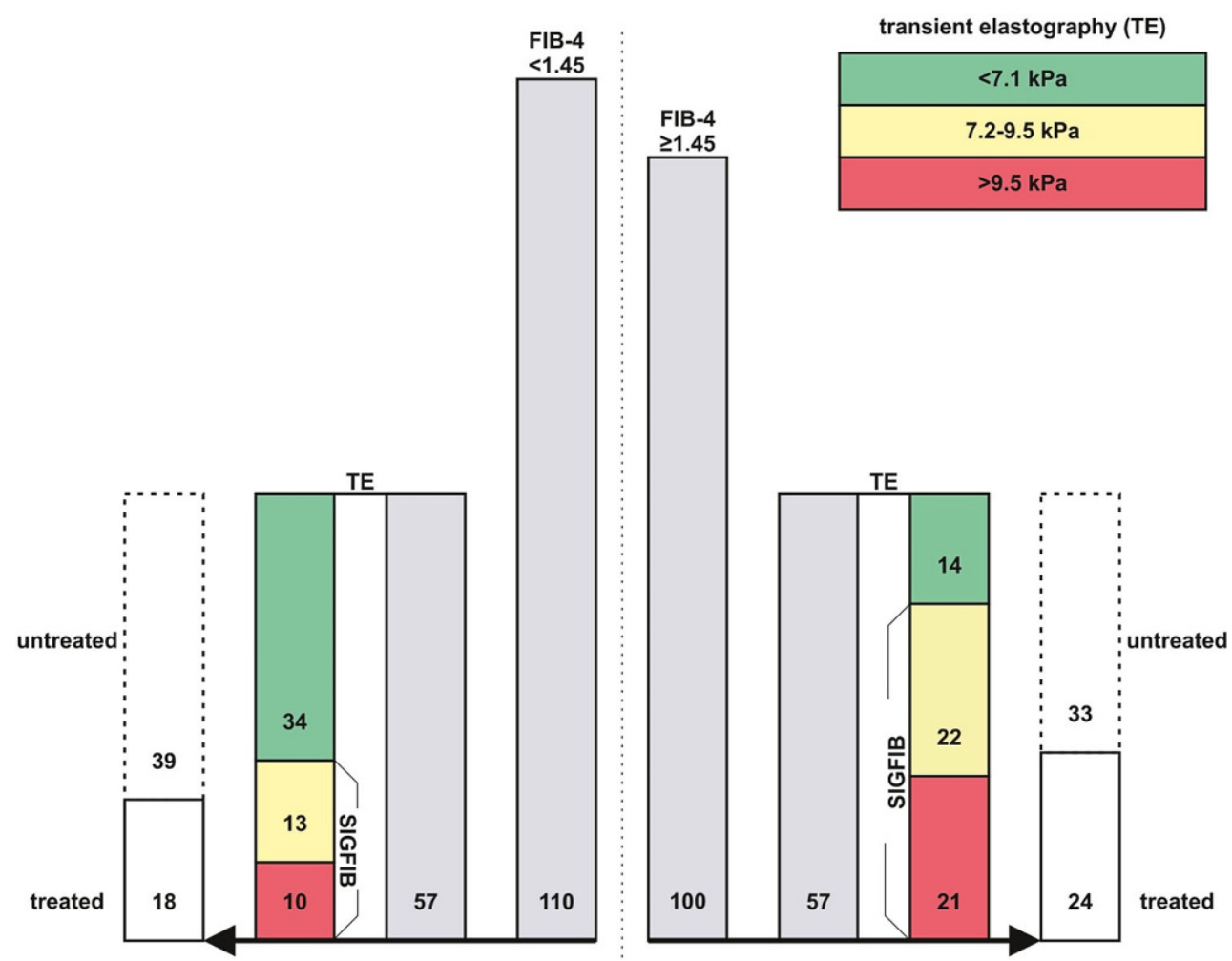

Mann-Whitney U-test was applied. Group comparisons of categorical variables were performed using the $\chi^{2}$-test and Fisher's exact test. A $P$ value of $\leq 0.05$ was considered statistically significant.

\section{Results}

\section{Patient characteristics}

Among the 1348 HIV-positive patients counselled by the HIV clinic, 33\% (439/1348) were HCV-antibody positive and $16 \%(210 / 1348)$ of patients had detectable HCV-RNA. The majority of HIV/HCV coinfected patients were male $(65 \%)$ and the median age was $37.9(\mathrm{SD} \pm 16.61)$ years. The main route of transmission was IVDA (75\%) followed by heterosexual intercourse $(14 \%)$ and $5 \%$ of patients were MSM. More than half of the patients were coinfected with HCV genotype 1 (57\%), while HCV genotypes 2, 3, and 4 were observed in 1, 10, and $32 \%$ of patients, respectively. Among HCV genotype 1 patients the subtype 1a (76\%) was more common than subtype $1 \mathrm{~b}$ $(24 \%)$. The vast majority of the patients were on ART (91\%), with $63 \%$ of HIV/HCV-coinfected patients having suppressed HIV-RNA $\left(<50\right.$ copies $\left.\mathrm{ml}^{-1}\right)$. The vast majority received at least one nucleoside reverse transcriptase inhibitor (N(t)RTI, 93\%), followed by HIV protease inhibitor (PI, 57\%) and non-nucleoside reverse transcriptase inhibitor (NNRTI, 18\%) treatment. Of the patients one quarter $(25 \%)$ received either an integrase inhibitor or an entry inhibitor (II/EI) as part of their ART.

\section{Comparison of patients with and without information on TE}

Of the HIV/HCV coinfected patients 114 (54\%) underwent TE, while 96 (46\%) patients did not (Table 1). There were a statistically significant differences in age $(41.5 \pm 11.0$ vs. $36.3 \pm 10.0$ years; $P<0.001)$ and patients who underwent TE showed a better immune status, i. e. a higher CD4+ T-lymphocyte count (511 (414) vs. 352 (380) cells $\mu \mathrm{l}^{-1} ; P=0.001$ ), proportion of patients with HIV-RNA <50 copies $\mathrm{ml}^{-1}(74 \%(84 / 113)$ vs. $49 \%(46 / 94) ; P<0.001)$ and HIV-RNA $<400$ copies $\mathrm{ml}^{-1}$ (89\% (100/113) vs. $\left.61 \%(57 / 94) ; P<0.001\right)$. Moreover, TE patients were more likely to receive an II/EI (35\% (38/108) vs. $11 \%(9 / 82) ; P<0.001)$. The opposite applied for HIV PI (44\% (48/108) vs. 73\% (60/82); $P<0.001)$. The proportion of patients with FIB- 4 index $\geq 1.45$ was comparable between patients with $(50 \%$ $(57 / 114))$ and without $(45 \%(43 / 96))$ information on TE $(P=0.452)$.

\section{Comparison of patients with FIB-4 index $<1.45$ and FIB-4 $\geq 1.45$}

Of the patients 110 had a FIB-4 index $<1.45$ and 100 patients presented with a FIB- 4 index $\geq 1.45$ (Supplementary Table 1; Fig. 1). Besides statistically significant differences in the variables included in the FIB-4 index (e.g. age, platelet count, AST and ALT), we observed a statistically significantly higher CD4+ T-lymphocyte count $\left(514\right.$ (426.8) vs. 353 (362) cells $\mu \mathrm{l}^{-1} ; P=$ 0.002 ) among patients with a FIB- 4 index $<1.45$. More- 
over, bilirubin was lower among patients with a FIB4 index $<1.45\left(0.43(0.34)\right.$ vs. $0.54(0.46) \mathrm{mg} \mathrm{dl}^{-1} ; P<$ 0.001). The proportion of patients who underwent $\mathrm{TE}$ was comparable between patients with a FIB-4 index $<1.45$ (52\% (57/110)) and $\geq 1.45$ (57\% (57/100); $P=$ 0.452).

Comparison of patients with FIB-4 index $<1.45$ and FIB-4 $\geq 1.45$ who underwent TE

A total of 57 patients (52\%) with a FIB- 4 index $<1.45$ as well as 57 patients (50\%) with a FIB- 4 index $\geq 1.45$ were evaluated by TE (Table 2; Fig. 1). Of the patients with a FIB-4 index $<1.45,40 \%(23 / 57)$ presented with a SIGFIB, and 18\% (10/57) even showed ADVFIB. Among patients FIB-4 index $\geq 1.45$, SIGFIB was observed in $86 \%(43 / 57 ; P=0.001$ when compared to FIB-4 index $<1.45)$ and ADVFIB in $37 \%(21 / 57 ; P=0.001$ when compared to FIB-4 index <1.45).

\section{Treatment initiation}

Among the patients who underwent TE, treatment was initiated in 37\% (42/114) (Table 3; Fig. 1). Although gender did not affect the probability of undergoing TE (Table 1), women were more likely to receive therapy. While $45 \%(19 / 42)$ of patients in whom treatment was initiated were female, $75 \%(54 / 72)$ of patients who were not treated were male $(P=0.026)$.

Unsurprisingly, the prevalence of SIGFIB $(88 \%$ (37/42) vs. $40 \%(29 / 72) ; P=0.001)$ and ADVFIB $(45 \%$ $(19 / 42)$ vs. $17 \%(12 / 72) ; P=0.001)$ were higher in patients in whom treatment was initiated.

Overall, treatment was initiated in only $20 \%(42 / 210)$ of a total of $210 \mathrm{HIV}$-positive patients with $\mathrm{CHC}$. The following interferon-free regimens were prescribed:

- Sofosbuvir (SOF)/ribavirin (RBV) - 5\% (2/42)

- SOF/daclatasvir (DCV) - 55\% (23/42)

- SOF/ledipasvir (LDV) - 29\% (12/42)

- Ombitasvir/paritaprevir/dasabuvir (3D) $\pm \mathrm{RBV}$ $12 \%(5 / 42)$

\section{Potential drug-drug interactions between directly acting antiviral agents}

The majority of HIV/HCV coinfected patients received ART $(91 \%)$. According to current data on drug-drug interactions (DDI) between DAA and ART [24], the combination of SOF/LDV or SOF/DCV would have been considered safe in all patients. A change in the ART regimen in patients with HCV genotypes 1 and 4 would have been necessary in $27 \%(31 / 113)$ and $68 \%$ (77/113) for the 2D/3D or the GPV/ELV regimens, respectively.

\section{Discussion}

Since HIV positive persons are at substantial risk of being coinfected with HCV due to the shared routes of transmission [2], the European AIDS Clinical Society recommends screening for HCV infection in HIVpositive persons on an annual basis [25]. The proportion of viremic HIV/HCV coinfected patients among all 1348 HIV-positive patients counselled by our HIV clinic was $16 \%(210 / 1348)$. The assessment of severity of liver fibrosis in HIV/HCV coinfected patients should be performed in all patients to determine urge for further treatment [15]; however, $46 \%$ of HIV/HCV coinfected patients did not undergo TE, and thus, had no access to modern IFN-free regimens, since until recently reimbursement of IFN-free treatment was primarily restricted to patients with SIGFIB in Austria.

We observed a significant difference in HIV infection parameters such as CD4+ T-lymphocyte count and HIV-RNA between patients who underwent TE compared to the ones who did not. According to previous studies [26, 27], immune status might be considered as a surrogate of adherence. Motivational barriers for ART treatment uptake and depression are considered as additional barriers to optimal adherence in HIV/HCV coinfected patients, when compared to HIV monoinfected patients [28]. Hence, the substantial proportion of $\mathrm{HIV} / \mathrm{HCV}$ coinfected patients without liver fibrosis assessment using TE might reflect the proportion of patients with limited compliance. This assumption is also supported by the statistically significantly lower proportion of patients on modern HIV integrase inhibitors in this group, since at our HIV clinic, HIV PI-based regimens are preferred in patients with suboptimal adherence.

Simple non-invasive indices have good diagnostic accuracy when combined with TE [29]. The FIB-4 is a non-invasive index for predicting ADVFIB which has initially been developed based on a cohort of HIV/HCV coinfected patients [18] and has been extensively validated in this setting [30-32]. Since FIB4 values were comparable between patients who underwent TE and patients who did not, we were able to assess whether the FIB-4 index allows identification of patients who are at risk for SIGFIB or ADVFIB, and thus, might have an urgent need for antiviral therapy.

A FIB-4 cut-off of 1.45 had a sensitivity of $68 \%(95 \%$ confidence interval, $95 \% \mathrm{CI}$ : $50-82 \%$ ) and negative predictive value (NPV) of $82 \%$ (95\%CI: $70-90 \%)$ for ruling out ADVFIB, which is numerically lower than the sensitivity $(70 \%)$ and NPV $(90 \%)$ reported in a previous study by Sterling et al. [18]. Similarly, specificity and PPV for ADVFIB (FIB-4 cut-off of 3.25) were numerically lower (93\% vs. $97 \%$ and $54 \%$ vs. $65 \%$, respectively), when compared to the study by Sterling et al. [18].Moreover, we aimed to assess whether the FIB-4 index allows identification of patients who are at risk for SIGFIB. Therefore, we stratified patients into two groups using a FIB-4 cut-off of 1.45. Specificity and 
original article

Table 2 Comparison of HIV/HCV-coinfected patients with FIB- $4<1.45$ and $\geq 1.45$ who underwent transient elastography

\begin{tabular}{|c|c|c|c|c|}
\hline Patient characteristics & $\begin{array}{l}\text { All patients } \\
(n=114)\end{array}$ & $\begin{array}{l}\text { FIB-4 }<1.45 \\
(n=57)\end{array}$ & $\begin{array}{l}\text { FIB- } 4 \geq 1.45 \\
(n=57)\end{array}$ & $P$-value \\
\hline \multicolumn{5}{|l|}{ Epidemiological characteristics } \\
\hline \multicolumn{5}{|l|}{ Sex } \\
\hline Male & $68 \%(77 / 114)$ & $72 \%(41 / 57)$ & $63 \%(36 / 57)$ & \multirow[t]{2}{*}{0.317} \\
\hline Female & $33 \%(37 / 114)$ & $28 \%(16 / 57)$ & $37 \%(21 / 57)$ & \\
\hline Age & $43.5(17.7)$ & $34.2(16.1)$ & $49.4(11.0)$ & $<0.001$ \\
\hline \multicolumn{5}{|l|}{ Transmission } \\
\hline MSM & $7 \%(8 / 114)$ & $5 \%(3 / 57)$ & $9 \%(5 / 57)$ & \multirow[t]{4}{*}{0.648} \\
\hline IVDU & $71 \%(81 / 114)$ & $72 \%(41 / 57)$ & $70 \%(40 / 57)$ & \\
\hline Heterosexual & $17 \%(19 / 114)$ & $19 \%(11 / 57)$ & $14 \%(8 / 57)$ & \\
\hline Others & $5 \%(6 / 114)$ & $4 \%(2 / 57)$ & $7 \%(4 / 57)$ & \\
\hline \multicolumn{5}{|l|}{ Laboratory parameters } \\
\hline Hemoglobin $\left(\mathrm{g} \mathrm{dl}^{-1}\right)$ & $13.8(2.85)$ & $13.9(2.32)$ & $13.8(2.62)$ & 0.375 \\
\hline Platelet count $\left(\mathrm{G} \mathrm{I}^{-1}\right)$ & $200 \pm 74.62$ & $241 \pm 63.8$ & $158 \pm 60.7$ & $<0.001$ \\
\hline White blood cell count $\left(\mathrm{GI}^{-1}\right)$ & $6.54 \pm 2.67$ & $7.28 \pm 2.11$ & $5.79 \pm 2.97$ & 0.003 \\
\hline Prothrombin time (\%) & $93.0(33.5)$ & $96.0(37.5)$ & $91.5(39.3)$ & 0.167 \\
\hline Albumin $\left(\mathrm{g} \mathrm{dl}^{-1}\right)$ & $42.9 \pm 4.66$ & $43.5 \pm 4.41$ & $42.3 \pm 4.86$ & 0.166 \\
\hline Creatinine $\left(\mathrm{mg} \mathrm{dl}^{-1}\right)$ & $0.84(0.24)$ & $0.80(0.24)$ & $0.88(0.23)$ & 0.205 \\
\hline Bilirubin $\left(\mathrm{mg} \mathrm{dl}^{-1}\right)$ & $0.50(0.46)$ & $0.43(0.34)$ & $0.53(0.46)$ & 0.011 \\
\hline AST $\left(\mathrm{UI}^{-1}\right)$ & $43.5(27.3)$ & $37.0(21.0)$ & $51.0(31.0)$ & $<0.001$ \\
\hline $\operatorname{ALT}\left(\mathrm{U} \mathrm{I}^{-1}\right)$ & $41.0(28.5)$ & $40.0(22.5)$ & $44.0(39.5)$ & 0.451 \\
\hline $\mathrm{GGT}\left(\mathrm{UI}^{-1}\right)$ & $76.0(86.0)$ & $62.5(75.8)$ & $90.0(80.0)$ & 0.002 \\
\hline \multicolumn{5}{|l|}{ HIV infection parameters } \\
\hline CD4+ T-lymphocyte count (cells $\mu$ I $\left.^{-1}\right)$ & $523 \pm 272$ & $628 \pm 279$ & $421 \pm 224$ & $<0.001$ \\
\hline HIV-RNA $<50$ copies $\mathrm{ml}^{-1}$ & $74 \%(84 / 113)$ & $75 \%(42 / 56)$ & $74 \%(42 / 57)$ & 0.873 \\
\hline HIV-RNA $<400$ copies $\mathrm{mr}^{-1}$ & $89 \%(100 / 113)$ & $86 \%(48 / 56)$ & $91 \%(78 / 57)$ & 0.358 \\
\hline CART & $95 \%(108 / 114)$ & $93 \%(53 / 57)$ & $97 \%(55 / 57)$ & 0.679 \\
\hline PI & $44 \%(48 / 108)$ & $45 \%(24 / 53)$ & $44 \%(24 / 55)$ & 0.863 \\
\hline $\mathrm{N}(\mathrm{t}) \mathrm{RTI}$ & $94 \%(102 / 108)$ & $96 \%(51 / 53)$ & $93 \%(51 / 55)$ & 0.679 \\
\hline NNRTI & $21 \%(23 / 108)$ & $21 \%(11 / 53)$ & $22 \%(12 / 55)$ & 0.893 \\
\hline II/EI & $35 \%(38 / 108)$ & $36 \%(19 / 53)$ & $35 \%(19 / 55)$ & 0.887 \\
\hline \multicolumn{5}{|l|}{ HCV infection parameters } \\
\hline HCV-RNA $\left(\log I U ~ m r^{-1}\right)$ & $6.00(1.07)$ & $5.80(1.23)$ & $6.24(0.99)$ & 0.72 \\
\hline HCV genotype & $94 \%(107 / 114)$ & $93 \%(53 / 57)$ & $95 \%(54 / 57)$ & - \\
\hline 1 & $56 \%(60 / 107)$ & $58 \%(31 / 53)$ & $54 \%(29 / 54)$ & \multirow[t]{4}{*}{0.145} \\
\hline 2 & $0 \%(0 / 107)$ & $0 \%(0 / 53)$ & $0 \%(0 / 54)$ & \\
\hline 3 & $32 \%(34 / 107)$ & $25 \%(13 / 53)$ & $39 \%(21 / 54)$ & \\
\hline 4 & $12 \%(13 / 107)$ & $17 \%(9 / 53)$ & $7 \%(4 / 54)$ & \\
\hline \multicolumn{5}{|l|}{ Liver stiffness } \\
\hline $\mathrm{F} 0 / \mathrm{F} 1(<7.1 \mathrm{kPa})$ & $42 \%(48 / 114)$ & $60 \%(34 / 57)$ & $25 \%(14 / 57)$ & $<0.001$ \\
\hline $\mathrm{F} 2(\geq 7.1$ and $<9.5 \mathrm{kPa})$ & $31 \%(35 / 114)$ & $23 \%(13 / 57)$ & $39 \%(22 / 57)$ & 0.068 \\
\hline $\mathrm{F} 3(\geq 9.5$ and $<12.5 \mathrm{kPa}$ ) & $7 \%(8 / 114)$ & $7 \%(4 / 57)$ & $7 \%(4 / 57)$ & 1 \\
\hline $\mathrm{F} 4(\geq 12.5 \mathrm{kPa})$ & $20 \%(23 / 114)$ & $11 \%(6 / 57)$ & $30 \%(17 / 57)$ & 0.010 \\
\hline Significant liver fibrosis ( $\geq 7.1 \mathrm{kPa}$ ) & $58 \%(66 / 114)$ & $40 \%(23 / 57)$ & $75 \%(43 / 57)$ & $<0.001$ \\
\hline Advanced liver fibrosis ( $\geq 9.5 \mathrm{kPa}$ ) & $27 \%(31 / 114)$ & $18 \%(10 / 57)$ & $37 \%(21 / 57)$ & 0.021 \\
\hline
\end{tabular}

$A L T$ alanine transaminase, AST aspartate transaminase, CART combined antiretroviral therapy, El entry inhibitors, GGT gamma-glutamyl transpeptidase, GT genotype, HCV hepatitis C virus, HIV human immunodeficiency virus, II integrase inhibitors, IVDU intravenous drug abuse, MSM men who have sex with men, NNRTI non-nucleoside reverse-transcriptase inhibitors, $N(t) R T / s$ nucleos(t)idic reverse transcriptase inhibitors, $P /$ protease inhibitor 
Table 3 Comparison of HIV/HCV coinfected patients who underwent transient elastography in whom HCV treatment was initiated vs. patients who did not undergo HCV treatment

\begin{tabular}{|c|c|c|c|c|}
\hline Patient characteristics & $\begin{array}{l}\text { All patients } \\
(n=114)\end{array}$ & $\begin{array}{l}\text { No treatment } \\
(n=72)\end{array}$ & $\begin{array}{l}\text { Treatment } \\
(n=42)\end{array}$ & $P$-value \\
\hline \multicolumn{5}{|l|}{ Epidemiological characteristics } \\
\hline \multicolumn{5}{|l|}{ Sex } \\
\hline Male & $68 \%(77 / 114)$ & $75 \%(54 / 72)$ & $55 \%(23 / 42)$ & \multirow[t]{2}{*}{0.026} \\
\hline Female & $33 \%(37 / 114)$ & $25 \%(18 / 72)$ & $45 \%(19 / 42)$ & \\
\hline Age & $43.5(17.7)$ & $41.0(16.3)$ & $46.75(16.9)$ & 0.078 \\
\hline \multicolumn{5}{|l|}{ Transmission } \\
\hline MSM & $7 \%(8 / 114)$ & $6 \%(4 / 72)$ & $10 \%(4 / 42)$ & \multirow[t]{4}{*}{0.468} \\
\hline IVDU & $71 \%(81 / 114)$ & $74 \%(53 / 72)$ & $67 \%(28 / 42)$ & \\
\hline Heterosexual & $17 \%(19 / 114)$ & $14 \%(10 / 72)$ & $21 \%(9 / 42)$ & \\
\hline Others & $5 \%(6 / 114)$ & $7 \%(5 / 72)$ & $2 \%(1 / 42)$ & \\
\hline \multicolumn{5}{|l|}{ Laboratory parameters } \\
\hline Hemoglobin $\left(\mathrm{g} \mathrm{dl}^{-1}\right)$ & $13.8(2.85)$ & $13.9(2.18)$ & $13.6(3.17)$ & 0.106 \\
\hline Platelet count $\left(\mathrm{GI}^{-1}\right)$ & $200 \pm 74.6$ & $206 \pm 66$ & $189 \pm 87.2$ & 0.232 \\
\hline White blood cell count $\left(\mathrm{GI}^{-1}\right)$ & $6.54 \pm 2.67$ & $6.76 \pm 2.67$ & $6.15 \pm 2.66$ & 0.238 \\
\hline Prothrombin time (\%) & $93(33.5)$ & $89(32.0)$ & $98(43.5)$ & 0.589 \\
\hline Albumin $\left(\mathrm{g} \mathrm{dl}^{-1}\right)$ & $42.91 \pm 4.66$ & $43.35 \pm 4.99$ & $42.14 \pm 3.95$ & 0.184 \\
\hline Creatinine $\left(\mathrm{mg} \mathrm{dl}^{-1}\right)$ & $0.84(0.24)$ & $0.81(0.18)$ & $0.90(0.35)$ & 0.102 \\
\hline Bilirubin $\left(\mathrm{mg} \mathrm{dl}^{-1}\right)$ & $0.50(0.46)$ & $0.50(0.33)$ & $0.52(0.58)$ & 0.920 \\
\hline AST $\left(\mathrm{U} \mathrm{I}^{-1}\right)$ & $43.5(27.3)$ & $47.0(33.0)$ & $39.5(25.3)$ & 0.203 \\
\hline $\operatorname{ALT}\left(\mathrm{U} \mathrm{I}^{-1}\right)$ & $41.0(28.5)$ & $45.0(36.8)$ & $37.5(24.8)$ & 0.102 \\
\hline GGT $\left(\mathrm{UI}^{-1}\right)$ & $76.0(86.0)$ & $77.5(86.3)$ & $72.0(83.0)$ & 0.960 \\
\hline \multicolumn{5}{|l|}{ HIV infection parameters } \\
\hline CD4+ T-lymphocyte count (cells $\mu$ F $\left.^{-1}\right)$ & $522.53 \pm 272.21$ & $514.76 \pm 256.03$ & $535.98 \pm 301.02$ & 0.693 \\
\hline HIV-RNA $<50$ copies $\mathrm{mI}^{-1}$ & $74 \%(84 / 113)$ & $72 \%(51 / 71)$ & $79 \%(33 / 42)$ & 0.428 \\
\hline HIV-RNA <400 copies $\mathrm{mr}^{-1}$ & $89 \%(100 / 113)$ & $86 \%(61 / 71)$ & $93 \%(39 / 42)$ & 0.264 \\
\hline CART & $95 \%(108 / 114)$ & $92 \%(66 / 72)$ & $100 \%(42 / 42)$ & 0.084 \\
\hline PI & $44 \%(48 / 108)$ & $53 \%(35 / 66)$ & $31 \%(13 / 42)$ & 0.024 \\
\hline$N(t) R T I$ & $94 \%(102 / 108)$ & $96 \%(63 / 66)$ & $93 \%(39 / 42)$ & 0.676 \\
\hline NNRTI & $21 \%(23 / 108)$ & $23 \%(15 / 66)$ & $19 \%(8 / 42)$ & 0.649 \\
\hline II/El & $35 \%(38 / 108)$ & $23 \%(15 / 66)$ & $55 \%(23 / 42)$ & 0.001 \\
\hline \multicolumn{5}{|l|}{ HCV infection parameters } \\
\hline HCV-RNA (log IU m $\left.r^{-1}\right)$ & $6.0(1.07)$ & $5.97(1.05)$ & $6.11(1.12)$ & 0.645 \\
\hline HCV genotype & $94 \%(107 / 114)$ & $90 \%(65 / 72)$ & $100 \%(42 / 42)$ & - \\
\hline 1 & $56 \%(60 / 107)$ & $57 \%(37 / 65)$ & $55 \%(23 / 42)$ & \multirow[t]{4}{*}{0.687} \\
\hline 2 & $0 \%(0 / 107)$ & $0 \%(0 / 65)$ & $0 \%(0 / 42)$ & \\
\hline 3 & $32 \%(34 / 107)$ & $29 \%(19 / 65)$ & $36 \%(15 / 42)$ & \\
\hline 4 & $12 \%(13 / 107)$ & $14 \%(9 / 65)$ & $9 \%(4 / 42)$ & \\
\hline \multicolumn{5}{|l|}{ FIB-4 } \\
\hline$<1.45$ & $50 \%(57 / 114)$ & $54 \%(39 / 72)$ & $43 \%(18 / 42)$ & 0.244 \\
\hline$\geq 1.45$ & $50 \%(57 / 114)$ & $46 \%(33 / 72)$ & $57 \%(24 / 42)$ & 0.244 \\
\hline \multicolumn{5}{|l|}{ Liver stiffness } \\
\hline $\mathrm{F} 0 / \mathrm{F} 1(<7.1 \mathrm{kPa})$ & $42 \%(48 / 114)$ & $60 \%(43 / 72)$ & $12 \%(5 / 42)$ & 0.001 \\
\hline $\mathrm{F} 2(\geq 7.1$ and $<9.5 \mathrm{kPa})$ & $31 \%(35 / 114)$ & $24 \%(17 / 72)$ & $43 \%(18 / 42)$ & 0.032 \\
\hline $\mathrm{F} 3$ ( $\geq 9.5$ and $<12.5 \mathrm{kPa}$ ) & $7 \%(8 / 114)$ & $3 \%(2 / 72)$ & $14 \%(6 / 42)$ & 0.028 \\
\hline $\mathrm{F} 4(\geq 12.5 \mathrm{kPa})$ & $20 \%(23 / 114)$ & $14 \%(10 / 72)$ & $31 \%(13 / 42)$ & 0.029 \\
\hline Significant liver fibrosis ( $\geq 7.1 \mathrm{kPa}$ ) & $58 \%(66 / 114)$ & $40 \%(29 / 72)$ & $88 \%(37 / 42)$ & 0.001 \\
\hline Advanced liver fibrosis ( $\geq 9.5 \mathrm{kPa}$ ) & $27 \%(31 / 114)$ & $17 \%(12 / 72)$ & $45 \%(19 / 42)$ & 0.001 \\
\hline
\end{tabular}

ALT alanine transaminase, AST aspartate transaminase, CART combined antiretroviral therapy, El entry inhibitors, GGT gamma-glutamyl transpeptidase, GT genotype, HCV hepatitis C virus, HIV human immunodeficiency virus, II integrase inhibitors, IVDU intravenous drug abuse, MSM men who have sex with men, NNRTI non-nucleoside reverse transcriptase inhibitors, N(t)RTIs nucleos(t)idic reverse transcriptase inhibitors, $P /$ protease inhibitor 
PPV for identifying patients with SIGFIB were substantially lower (vs. ADVFIB) with 57 and 37\%, respectively. Importantly, FIB- $4<1.45$ did not rule out SIGFIB or ADVFIB, since $40 \%$ of patients with a FIB$4<1.45$ had SIGFIB and $18 \%$ even had ADVFIB. Thus, using the previously established FIB- 4 cut-off of 1.45 , FIB-4 index is not useful for prescreening $\mathrm{HIV} / \mathrm{HCV}-$ coinfected patients for SIGFIB or ADVFIB.

Our analysis revealed that treatment was initiated in only $20 \%$ of all HIV/HCV coinfected patients. Modern IFN-free regimens have shown excellent results in clinical trials with SVR rates exceeding 95\% [10-14]. Although the extensive inclusion and exclusion criteria of clinical trials have raised concerns about the generalizability of these findings [33], real-life studies have shown encouraging results in unselected patients [34-36]. Thus, considering the excellent efficacy and safety of modern regimens, it is essential to improve treatment uptake rates to reduce the burden of HCV-related advanced liver disease [34]. Particularly people who inject drugs [2] need to be addressed by customized concepts. A higher knowledge of HCV is associated with increased willingness for HCV treatment [37] and thus a general lack of awareness of HCV is still a major concern [38]. Moser et al. [39] recently described a promising approach to address patients who are on opioid substitution therapy. In order to receive opioid substitution, a visit in a low-threshold drug treatment facility was mandatory on a daily basis, allowing HCV treatment to be coadministered. This approach substantially improved adherence [39]. Moreover, HCV treatment outcomes are not affected by opioids [40]. Thus, considering the recently extended access to IFN-free regimens, such programs might decrease the incidence of $\mathrm{HCV}$ among patients with IVDA [41].

Patients coinfected with HIV/HCV are no longer considered as difficult to treat population [5]; however, DDIs are a major concern when prescribing DAA-based regimens, especially, when regimens including a HCV PI such as the $2 \mathrm{D} / 3 \mathrm{D}$ regimen or GPV and ELV are used [15]. Our findings suggest that combinations of SOF/LDV or SOF/DCV could be prescribed safely with any ART used in our cohort. In contrast, a change in the ART regimen would be necessary in $27 \%$ for the $2 \mathrm{D} / 3 \mathrm{D}$ regimen and in $68 \%$ if GPV/ELV are prescribed. In these cases, ART treatment options would be limited to NRTIs and II/EIs [15]. With respect to the common use of HIV PIs as part of ART, physicians would have to deal with additional difficulties. Since PIs appear to have a higher resistance barrier than IIs, ART treatment history needs to be evaluated carefully to avoid virologic failure [42].

Although IFN-free regimens are now reimbursed for most HIV-positive patients with $\mathrm{CHC}$ regardless of the severity of liver fibrosis, considerable compliance issues remain. Thus, it is unclear whether extending the reimbursement of IFN-free treatment to patients without SIGFIB will lead to the anticipated increase in treatment uptake rates. Additional studies are needed to investigate the underlying factors hindering liver fibrosis assessment and treatment uptake in order to promote HCV elimination in this special population.

In conclusion, FIB-4 was not useful for ruling out ADVFIB in our cohort of HIV/HCV. Treatment was initiated only in a small proportion of $\mathrm{HIV} / \mathrm{HCV}$ during the first 2 years of IFN-free treatment availability, although the observed proportion $(20 \%)$ of patients with SIGFIB (assessed by TE) was considerably higher (58\%). Thus, it requires the ongoing combined efforts of both HIV and HCV specialists to increase treatment uptake rates in this special population.

Acknowledgments Open access funding provided by Medical University of Vienna.

Funding Open access funding provided by Medical University of Vienna.

\section{Compliance with ethical guidelines}

Conflict of interest P. Schwabl received payments for lectures from Boehringer Ingelheim and Roche and travel support from AbbVie, Gilead, and Roche. T. Bucsics received payments for lectures from Roche and travel support from Bristol-Myers Squibb. B. Scheiner received travel support from Gilead. M.C. Aichelburg received honoraria for consulting from Gilead and MSD and travel support from AbbVie, Gilead, and MSD. K. Grabmeier-Pfistershammer received honoraria for consulting from Gilead, payments for lectures from Bristol-Myers Squibb and ViiV, as well as travel support from Bristol-Myers Squibb, Gilead, and GlaxoSmithKline. M. Trauner received grants from MSD, honoraria for consulting from AbbVie, Gilead, Janssen, and MSD, payments for lectures from Gilead, MSD, and Roche, as well as travel support from Gilead. M. Peck-Radosavljevic received grants from Gilead, MSD, and Roche, honoraria for board membership and consulting from AbbVie, Boehringer Ingelheim, BristolMyers Squibb, Gilead, Janssen, and MSD, as well as payments for lectures from AbbVie, Boehringer Ingelheim, Bristol-Myers Squibb, Gilead, Janssen, MSD, and Roche. T. Reiberger received payments for lectures from Roche and MSD, as well as travel support from Gilead, MSD, and Roche; TR serves as a consultant for Gilead, Abbvie and MSD. M. Mandorfer received honoraria for consulting from Janssen, payments for lectures from Boehringer Ingelheim, Bristol-Myers Squibb, Janssen, and Roche, as well as travel support from AbbVie, Gilead, MSD, and Roche. D. Chromy, R. Strassl and F. Mayer declare that they have no competing interests.

Ethical standards This study was conducted in accordance with the Declaration of Helsinki and was approved by the local ethics committee of the Medical University of Vienna (EK Nr. 1699/2013).

Open Access This article is distributed under the terms of the Creative Commons Attribution 4.0 International License (http://creativecommons.org/licenses/by/4.0/), which permits unrestricted use, distribution, and reproduction in any medium, provided you give appropriate credit to the original author(s) and the source, provide a link to the Creative Commons license, and indicate if changes were made. 


\section{References}

1. Gower E,Estes C, BlachS, Razavi-Shearer K, RazaviH.Global epidemiology and genotype distribution of the hepatitis $\mathrm{C}$ virus infection. JHepatol. 2014;61 (1 Suppl):S45-S57.

2. Alter MJ. Epidemiology of viral hepatitis and HIV co-infection. JHepatol. 2006;44(1 Suppl):S6-S9.

3. Sulkowski MS, Thomas DL. Hepatitis C in the HIV-infected person. Ann Intern Med. 2003;138(3):197-207.

4. Sherman KE, Rouster SD, Chung RT, Rajicic N. Hepatitis $\mathrm{C}$ virus prevalence among patients infected with human Immunodeficiency virus: a cross-sectional analysis of the US adult AIDS clinical trials group. Clin Infect Dis. 2002;34(6):831-7.

5. Mandorfer M, Schwabl P, Steiner S, Reiberger T, PeckRadosavljevic M.Advances in the management of HIV/HCV coinfection. Hepatol Int. 2016;10(3):424-35. doi:10.1007/ s12072-015-9691-4.

6. Thomson EC, Fleming VM, Main J, Klenerman P, Weber J, Eliahoo J, et al. Predicting spontaneous clearance of acute hepatitis $\mathrm{C}$ virus in a large cohort of HIV-1-infected men. Gut. 2011;60(6):837-45.

7. Graham CS, Baden LR, Yu E, Mrus JM, Carnie J, Heeren T, et al. Influence of human immunodeficiency virus infection on the course of hepatitis $C$ virus infection: a meta-analysis. Clin Infect Dis. 2001;33(4):562-9.

8. Smith C, Sabin CA, Lundgren JD, Thiebaut R, Weber R, Law $\mathrm{M}$, et al. Factors associated with specific causes of death amongst HIV-positive individuals in the D:A:D study. AIDS. 2010;24(10):1537-48.

9. Molina JM, Orkin C, Iser DM, Zamora FX, Nelson M, Stephan C, et al. Sofosbuvir plus ribavirin for treatment of hepatitis $\mathrm{C}$ virus in patients co-infected with HIV (PHOTON-2): a multicentre, open-label, non-randomised, phase 3 study. Lancet. 2015;385(9973):1098-106.

10. Wyles DL, Ruane PJ, Sulkowski MS, Dieterich D, Luetkemeyer A, Morgan TR, et al. Daclatasvir plus Sofosbuvir for HCV in patients coinfected with HIV-1. N Engl J Med. 2015;373(8):714-25.

11. Osinusi A, Townsend K, Kohli A, Nelson A, Seamon C, Meissner EG, et al. Virologic response following combined ledipasvir and sofosbuvir administration in patients with HCV genotype 1 and HIV co-infection. JAMA. 2015;313(12):1232-9.

12. Sulkowski MS, Eron JJ, Wyles D, Trinh R, Lalezari J, Wang $\mathrm{C}$, et al. Ombitasvir, paritaprevir co-dosed with ritonavir, dasabuvir, and ribavirin for hepatitis $C$ in patients co-infected with HIV-1: a randomized trial. JAMA. 2015;313(12):1223-31.

13. Sulkowski M, Hezode C, Gerstoft J, Vierling JM, Mallolas J, Pol S, et al. Efficacy and safety of 8 weeks versus 12 weeks of treatment with grazoprevir (MK-5172) and elbasvir (MK8742) with or without ribavirin in patients with hepatitis $\mathrm{C}$ virus genotype 1 mono-infection and HIV/hepatitis $\mathrm{C}$ virus co-infection (C-WORTHY): a randomised, open-label phase 2 trial. Lancet. 2015;385(9973):1087-97.

14. Rockstroh JK, Nelson M, Katlama C, Lalezari J, Mallolas J, Bloch M, et al. Efficacy and safety of grazoprevir (MK-5172) andelbasvir (MK-8742) in patientswithhepatitisCvirus and HIV co-infection (C-EDGE CO-INFECTION): a non-randomised, open-label trial. LancetHIV.2015;2(8):e319-e27.

15. European Association for the Study of the Liver. EASL Recommendations on Treatment of Hepatitis C2016. JHepatol. 2017;66(1):153-94.

16. Sozialversicherungsträger Hdö. Hochpreisige Medikamenteund steigendeArbeitslosigkeitlassen Krankenkassen heuer in die roten Zahlen rutschen 2015. http://www.hauptverband.at/portal27/portal/hvbportal/ content/contentWindow;jsessionid=34950CA18AD908A7 1B4F4B31ECD080A5.jbport_271_esvportal_a?contentid= 10007.754367\&amp;action=2\&amp; viewmode=content. Accessed March 6th 2017.

17. de Ledinghen V, Douvin C, Kettaneh A, Ziol M, Roulot D, Marcellin P, et al. Diagnosis of hepatic fibrosis and cirrhosis by transient elastography in HIV/hepatitis C virus-coinfected patients. J Acquir Immune Defic Syndr. 2006;41(2):175-9.

18. Sterling RK, Lissen E, Clumeck N, Sola R, Correa MC, Montaner J, et al. Development of a simple noninvasive index to predict significant fibrosis in patients with $\mathrm{HIV} / \mathrm{HCV}$ coinfection. Hepatology. 2006;43(6):1317-25.

19. Castera L, Sebastiani G, Le Bail B, de Ledinghen V, Couzigou P, Alberti A. Prospective comparison of two algorithms combining non-invasive methods for staging liver fibrosis in chronichepatitis C. J Hepatol. 2010;52(2):191-8.

20. Reiberger T, Ferlitsch A, Payer BA, Pinter M, Homoncik M, Peck-Radosavljevic M, et al. Non-selective beta-blockers improve the correlation of liver stiffness and portal pressure in advanced cirrhosis. J Gastroenterol. 2012;47(5):561-8.

21. Schwabl P, Bota S, Salzl P, Mandorfer M, Payer BA, Ferlitsch A, et al. New reliability criteria for transient elastography increase the number of accurate measurements for screening of cirrhosis and portal hypertension. Liver Int. 2015;35(2):381-90.

22. Castera L, Vergniol J, Foucher J, Le Bail B, Chanteloup E, Haaser M, et al. Prospective comparison of transient elastography, fibrotest, APRI, and liver biopsy for the assessment of fibrosis in chronic hepatitis C. Gastroenterology. 2005;128(2):343-50.

23. Vallet-Pichard A, Mallet V, Nalpas B, Verkarre V, Nalpas A, Dhalluin-VenierV, et al. FIB-4: an inexpensive and accurate marker of fibrosis in HCV infection. comparison with liver biopsy and fibrotest. Hepatology. 2007;46(1):32-6.

24. Liverpool TUo. HEP Drug Interaction 2016. http://www. hep-druginteractions.org/. Accessed September 8th 2016

25. Lundgren J, Ryom L. et al. EACS Guidelines. Brussels: European AIDS Clinical Society (EACS);2015.

26. Inciardi JF, Leeds AL. Assessing the utility of a community pharmacy refill record as a measure of adherence and viral load response in patients infected with human immunodeficiency virus. Pharmacotherapy. 2005;25(6):790-6.

27. San Lio MM, Carbini R, Germano P, Guidotti G, Mancinelli S, Magid NA, et al. Evaluating adherence to highly active antiretroviral therapy with use of pill counts and viral load measurement in the drug resources enhancement against AIDS and malnutrition program in Mozambique. Clin Infect Dis. 2008;46(10):1609-16.

28. Shuper PA, Joharchi N, Irving H, Fletcher D, Kovacs C, Loutfy $\mathrm{M}$, et al. Differential predictors of ART adherence among HIV-monoinfected versus HIV/HCV-coinfected individuals. AIDSCare. 2016;28(8):954-62.

29. European Association for Study of Liver, Asociacion Latinoamericana para el Estudio del Higado.. EASL-ALEH clinical practice guidelines: non-invasive tests for evaluation of liver disease severity and prognosis. J Hepatol. 2015;63(1):237-64.

30. Tural C, Tor J, Sanvisens A, Perez-Alvarez N, Martinez E, Ojanguren I, et al. Accuracy of simple biochemical tests in identifying liver fibrosis in patients co-infected with human immunodeficiency virus and hepatitis $C$ virus. Clin Gastroenterol Hepatol. 2009;7(3):339-45.

31. Shah AG, Smith PG, Sterling RK. Comparison of FIB-4 and APRI in HIV-HCV coinfected patients with normal and elevated ALT. Dig Dis Sci. 2011;56(10):3038-44. 
32. Gounder PP, Haering C, Bruden DJ, Townshend-Bulson L, Simons BC, Spradling PR, et al. Does incorporating change in APRI or FIB-4 indices over time improve the accuracy of a single index for identifying liver fibrosis in persons with chronic hepatitis C virus infection? J Clin Gastroenterol. 2016; doi:10.1097/MCG.0000000000000753.

33. Zoulim F, Liang TJ, Gerbes AL, Aghemo A, Deuffic-Burban S, Dusheiko G, et al. Hepatitis C virus treatment in the real world: optimising treatment and access to therapies. Gut. 2015;64(11):1824-33.

34. Welzel TM, Petersen J, Herzer K, Ferenci P, Gschwantler $\mathrm{M}$, Wedemeyer $\mathrm{H}$, et al. Daclatasvir plus sofosbuvir, with or without ribavirin, achieved high sustained virological response rates in patients with HCVinfection and advanced liver disease in a real-world cohort. Gut. 2016; doi:10.1136/ gutjnl-2016-312444.

35. Steiner S, Bucsics T, Schwabl P, Mandorfer M, Scheiner B, Aichelburg MC, et al. Progress in eradication of HCV in HIV positive patients with significant liver fibrosis in Vienna. Wien Klin Wochenschr. 2017; doi:10.1007/s00508-0161162-y.

36. Mandorfer M, Schwabl P, Steiner S, Scheiner B, Chromy D, Bucsics T, et al. Interferon-free treatment with sofosbuvir/ daclatasvir achieves sustained virologic response in $100 \%$ of HIV/hepatitis Cvirus-coinfected patients with advanced liver disease. AIDS. 2016;30(7):1039-47.
37. Mah A, Hull MW, DeBeck K, Milloy MJ, Dobrer S, Nosova E, et al. Knowledge of hepatitis $\mathrm{C}$ and treatment willingness amongst people who inject drugs in an era of direct acting antivirals. Int J Drug Policy. 2017; doi:10.1016/j.drugpo. 2017.02.006.

38. Muhlberger N, Schwarzer R, Lettmeier B, Sroczynski G, Zeuzem S, Siebert U. HCV-related burden of disease in Europe: a systematic assessment of incidence, prevalence, morbidity, and mortality. BMC Public Health. 2009;9:34.

39. Moser S, Schütz A, Marchart K, Ambrosch S, Karpi A, Gutic E, et al. Direct observed therapy of chronic hepatitis $C$ with interferon-free all-oral regimens at a low-threshold drug treatment facility - a new concept for treatment of patients with borderline compliance receiving Opioid substitution therapy. JHepatol. 2016;64(2):S822.

40. Butner JL, Gupta N, Fabian C, Henry S, Shi JM, Tetrault JM. Onsite treatment of HCV infection with direct acting antivirals within an opioid treatment program. J Subst Abuse Treat. 2017;75:49-53.

41. Cousien A, Leclerc P, Morissette C, Bruneau J, Roy E, Tran VC, et al. The need for treatment scale-up to impact HCV transmission in people who inject drugs in Montreal, Canada: a modelling study. BMCInfect Dis. 2017;17(1):162. 42. Van den Eynde E, Podzamczer D. Switch strategies in antiretroviral therapy regimens. Expert Rev Anti Infect Ther. 2014;12(9):1055-74. 